\title{
Evolution of Courtship Songs in Xenopus: Vocal Pattern Generation and Sound Production
}

\author{
Elizabeth C. Leininger ${ }^{a}$ Darcy B. Kelley ${ }^{b}$ \\ ${ }^{a}$ Biology Department and Cross-Disciplinary Neurosciences Program, St. Mary's College of Maryland, \\ St. Mary's City, Md., and ' Program in Neurobiology and Behavior and Department of Biological Sciences, \\ Columbia University, New York, N.Y., USA
}

\section{Key Words}

Central pattern generator · Evolution · Larynx · Sex

differences - Species differences - Vocalization $\cdot$ Xenopus

\begin{abstract}
The extant species of African clawed frogs (Xenopus and Silurana) provide an opportunity to link the evolution of vocal characters to changes in the responsible cellular and molecular mechanisms. In this review, we integrate several robust lines of research: evolutionary trajectories of Xenopus vocalizations, cellular and circuit-level mechanisms of vocalization in selected Xenopus model species, and Xenopus evolutionary history and speciation mechanisms. Integrating recent findings allows us to generate and test specific hypotheses about the evolution of Xenopus vocal circuits. We propose that reduced vocal sex differences in some Xenopus species result from species-specific losses of sexually differentiated neural and neuromuscular features. Modification of sex-hormone-regulated developmental mechanisms is a strong candidate mechanism for reduced vocal sex differences.

(c) 2015 S. Karger AG, Basel
\end{abstract}

The extant species of African clawed frogs (Xenopus and Silurana) provide an opportunity to link the evolution of vocalizations to changes in the responsible cellular and molecular mechanisms. African clawed frogs exhibit rich vocal repertoires, which include vocalizations that are diverse and species-specific (e.g. male advertisement calls) [Tobias et al., 2011] as well as vocalizations that are more similar across species (e.g. release calls) [Tobias et al., 2014]. Phylogenetic surveys of these vocalizations have revealed evolutionary patterns in call characters. Our understanding of the physiological bases of vocal production is largely due to 2 advantageous ex vivo neurophysiological preparations: the isolated brain that produces vocal nerve activity patterns that match actual calls [Rhodes et al., 2007] and the isolated vocal organ (larynx) that produces sound pulses in response to nerve activity [Tobias and Kelley, 1987]. In Xenopus laevis, for example, activity patterns recorded from the vocal nerve during calling exactly match the sound pulse patterns that comprise sex-specific vocalizations [Yamaguchi and Kelley, 2000]. The vocal patterns themselves arise from a vocal pattern generator (VPG) within the hindbrain [Rhodes et al., 2007; Zornik and Kelley, 2007]. The isolated larynx is a useful preparation for understanding how activity of the vocal motor nerve is transduced into sound; it produces

Elizabeth C. Leininger

Biology Department and Cross-Disciplinary Neurosciences Program

St. Mary's College of Maryland

19852 E. Fisher Rd., St. Mary's City, MD 20686-3001 (USA)

E-Mail ecleininger@smcm.edu 
muscle contractions and actual sound pulses ex vivo in response to laryngeal nerve rootlet stimulation [Tobias and Kelley, 1987]. These preparations have led to the identification of cellular mechanisms of vocalization, both between sexes and, increasingly, between species, the 2 foci of this review.

\section{Vocalizations in the Xenopodinae}

African clawed frogs (Xenopus and Silurana) are aquatic anurans (Pipidae) native to sub-Saharan Africa [Kobel et al., 1996]. As for other anurans [Gerhardt and Huber, 2002], vocal interactions are key for reproduction. The basic unit of vocalization is a sound pulse, produced by the larynx in response to activity in the vocal motor nerve [Tobias and Kelley, 1987]. These sound pulses occur in bouts (trains of pulses separated by interpulse intervals, IPIs) that vary in temporal features by species, sex, and reproductive condition.

\section{The Vocal Repertoire}

The vocal repertoire is most completely outlined for $X$. laevis [for review see Zornik and Kelley, 2011] and is illustrated in figure 1. Each call type is specific to a social context, varies with reproductive state and, in the laboratory, can be evoked either in isolation, in opposite-sex, or in same-sex pairs. The ticking vocalization (fig. 1A) is produced by sexually unreceptive females when clasped by males; rapping (fig. 1B) is produced by gravid females in response to male advertisement calling (fig. 1C) [Tobias et al., 1998]. The male advertisement call is produced in isolation or with a conspecific of either sex [Tobias et al., 2011]. Ticking transiently silences advertising males [Elliott and Kelley, 2007], while rapping evokes the answer call (fig. 1D) [Tobias et al., 1998]. When a male clasps another frog, he produces the amplectant call (fig. 1E). A clasped male produces a growl (release call; fig. 1F) [Tobias et al., 2004]. Interactions between male pairs also include vocal dominance produced by the advertisement call; the subordinated male exhibits prolonged vocal suppression (relative to ticking-induced vocal suppression) [Tobias et al., 2010]. Vocal interactions between pairs of frogs (e.g. chirp/ growl, rapping/answer calling) have the characteristics of duets [Tobias et al., 1998, 2010]. While the mechanism of vocal production (described below) does not produce any visible movements of the throat or body wall in either sex, male and female sound pulses can be distinguished by their frequencies and IPIs [Tobias et al., 1998], and the vocalizing individual can be identified using a small, relatively insensitive hydrophone [Tobias et al., 2004].

In all species, males produce an advertisement call and give a release call when clasped; in some species, females also produce a release call [Tobias et al., 2011, 2014]. The vocal repertoire has otherwise not been completely described for species other than $X$. laevis and X. borealis. $X$. borealis males produce a distinctive call type when approaching another frog [Yager, 1992a], suggesting that its repertoire extends beyond advertisement and release calls. Yager [1992a] also described vocal suppression in interacting pairs of $X$. borealis males. He noted that the release call (termed the 'agonistic call') sometimes occurred in response to the advertisement call, but not necessarily during physical contact.

\section{Advertisement Calls and Release Calls; Comparative Acoustic Features}

Effective social interactions rely on communication of the signaler's species, sex, and reproductive state. For example, calls of conspecific males are more effective in attracting females than those of heterospecific males [Picker, 1983]. In principle, the spectral (sound frequency components) and/or temporal (pattern of pulses) features of advertisement calls could serve as reliable indicators of sex and species identity during social encounters. In all but one (X. fraseri) species of Xenopus, male advertisement call sound pulses include 2 dominant frequencies [Tobias et al., 2011]. Both the lower frequency (DF1) and the higher frequency (DF2) overlap extensively across species. For example, DF2 is the same in $X$. itombwensis as it is in the distantly related $X$. borealis; $X$. laevis and $X$. lenduensis (also distantly related) share DF1. Thus, neither DF1 nor DF2 alone are reliable indicators of species identity [Tobias et al. 2011].

Temporal patterns of male advertisement calls vary markedly even between closely related species (fig. 2). These patterns can be sorted into 4 groups: click-type, burst-type, trill-type, and biphasic-type (fig. 2A). Bursts and trills (mono- or biphasic) are intensity-modulated: pulse intensity increases as the call progresses. While call patterns themselves can be shared by distantly related species in separate clades, the combination of call characters - call type, IPI, call duration, inter-call interval, and intensity modulation - is species-specific [Tobias et al., 2011]. These species-specific call characters are determined by a set of neural circuits in the hindbrain, collectively known as the VPG [Rhodes et al., 2007] and by the larynx. 


\section{Sex $q$}

A

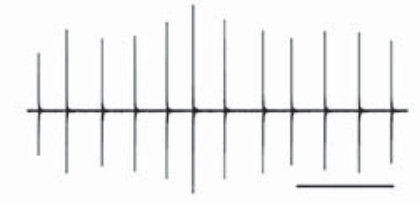

B

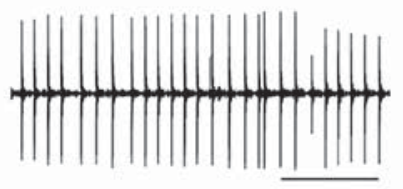

C

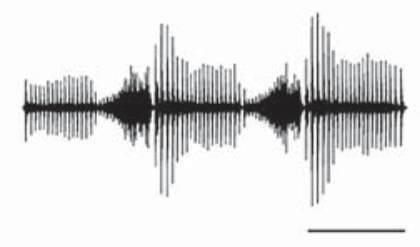

D

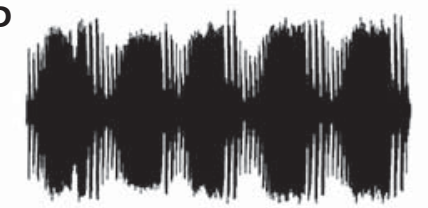

E

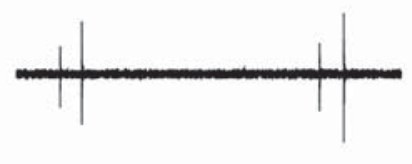

$\mathbf{F}$

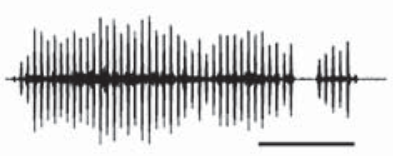

G

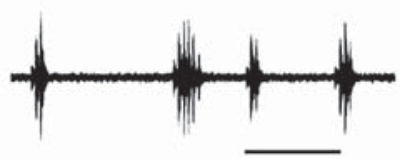

Social context
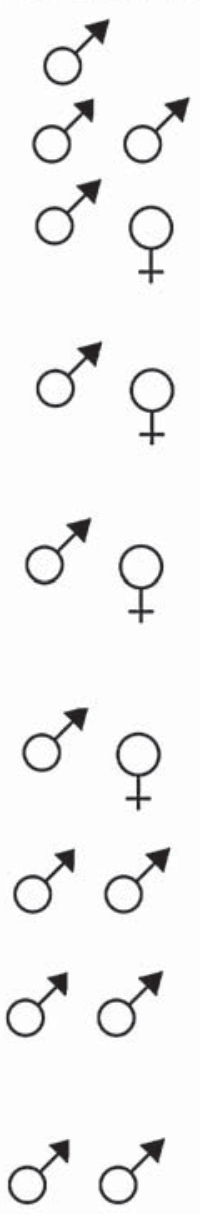

Fig. 1. The sex-specific vocal repertoire of $X$. laevis illustrated as oscillograms (sound intensity versus time). A Female ticking. B Female rapping. C Male advertisement calling. D Male answer calling. E Male amplectant calling. F Male growling. G Male chirping. Scale bars are $500 \mathrm{~ms}$ except for $\mathbf{F}$ and $\mathbf{G}(250 \mathrm{~ms})$. Calls are made up of trains of sound pulses that form distinctive temporal patterns. The male advertisement and answer calls include fast and slow trill portions and are thus biphasic; other calls are monopha-

In contrast, male and female release calls vary little across species and sexes [Tobias et al., 2014]. Though clasp duration can be longer for male/female than for male/male pairs, release calls do not provide the clasping sic. Advertisement and answer calls are intensity-modulated; other calls are not. Though shown here normalized to maximum pulse intensity for clarity, some calls are loud (advertisement calls and rapping, for example) and some are soft (ticking and amplectant calls). The male advertisement call is given when alone or in the presence of conspecifics of either sex. Other call types are context specific and depend on the sex and reproductive state of the partner (social context). Modified from Zornik and Kelley [2011]. 


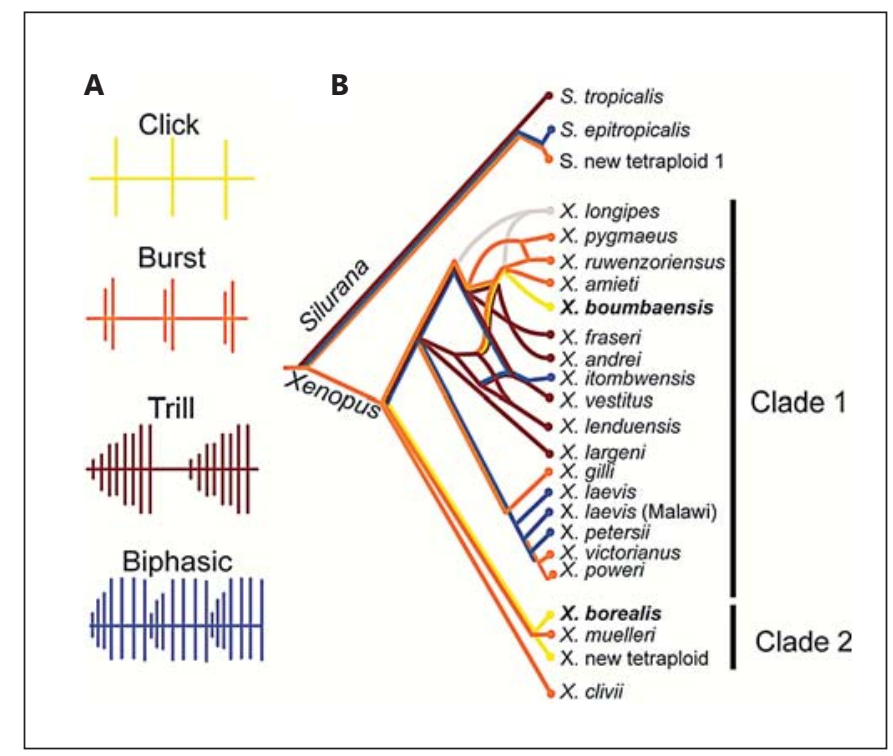

Fig. 2. A Male advertisement call temporal patterns range in complexity from single sound pulses repeated at long intervals (click patterns) to temporally complex biphasic calls. B Mapping of advertisement calls onto the molecular phylogeny of the Xenopodinae reveals that advertisement call patterns are homoplasious. For example, burst-type calls occur in all branches of the phylogeny. A parsimony analysis suggests that the moderately complex bursttype advertisement call structure is ancestral. This figure is reproduced from Leininger and Kelley [2013].

er than frequencies in the advertisement call. This feature gave rise to the name for male release calls in X. laevis: growling [Picker, 1983].

What are the relative contributions of temporal and spectral features to vocal recognition? Playbacks of rapping, the female receptive call, induce males to approach the speaker and to change from advertisement to answer calling (fig. 1) [Tobias et al., 1998]. Playbacks of the female unreceptive call, ticking, suppress male calling. While spectral properties of the sound pulses in rapping and ticking do not differ, the mean IPI in rapping is about half of that for ticking [Tobias et al., 1998]. Playbacks of rapping calls constructed from ticking sound pulses are effective in stimulating male calling, suggesting that temporal features predominate when males distinguish receptive from unreceptive calls [Tobias et al., 1998]. The effective temporal parameters for recognition of rapping and ticking have been defined [Elliott and Kelley, 2007]. Vignal and Kelley [2007] also used playbacks to determine whether rapping constructed of sound pulses with male-like spectral features would be as effective in evoking male calling as rapping constructed with sound puls-

Evolution of Courtship Songs in Xenopus es with female-like spectral features. Modifying the temporal pattern of rapping significantly decreased the proportion of the time spent advertising to playbacks, suggesting that males decode rapping using temporal information. Modifying the spectral domain of rapping (for example, to white-noise or male-typical frequencies) while keeping the temporal domain constant did not change the proportion of time males spent advertising to playback, but it did reduce the amount of answer calling to playback. These results suggest that spectral information may additionally contribute to evaluation of a female's receptive state.

\section{Evolution of Call Patterns}

\section{Phylogeny}

In Xenopus species, chromosome numbers are multiples of 18; the presumed diploid ancestor is not represented, to date, in an extant species. Two Xenopus clades can be distinguished (fig. 2) [Bewick et al., 2011]. Clade 1 includes species such as $X$. laevis, $X$. victorianus, and $X$. poweri, which are all tetraploid (36 chromosomes). It also includes a group of higher ploidy species, such as $X$. boumbaensis (octoploid) whose origins are ascribed to further interspecific hybridization and allopolyploidizations [reviewed in Evans, 2008]. This portion of the phylogeny thus has a reticulated character. Clade 2 includes 3 tetraploid species: $X$. borealis, $X$. muelleri, and an additional undescribed tetraploid [Bewick et al., 2011]. The phylogenetic affinity of the tetraploid X. clivii is as yet unresolved, with different strongly supported affinities to Clade 1 or 2 recovered by analysis of mitochondrial DNA or the linked autosomal genes RAG1 and RAG2, respectively [Evans et al., 2004, 2005; Evans, 2007].

The sister clade to Xenopus, Silurana (fig. 2), includes 1 species with 20 chromosomes and at least 2 species with 40 chromosomes [Evans, 2008]. The 20-chromosome species (S. tropicalis) is believed to have descended from a diploid most recent common ancestor (MRCA), and the 40-chromosome species are believed to represent allopolyploidy resulting from ancient hybridization [Evans, 2008]. The differences in chromosome number (20 vs. 18) appear due to chromosome fusion in the MRCA of Xenopus and Silurana [Hellsten et al., 2010].

\section{Advertisement Calls}

A parsimony analysis of call pattern mapped onto the phylogeny suggests that both the simplest (click-type) and the most complex (biphasic-type) male advertise- 
ment call patterns are derived from an ancestral bursttype pattern [Tobias et al., 2011]. The click-type pattern occurs in both clades but is rare (3 species in yellow; fig. 2B). The distribution of the click-type advertisement call pattern allows us to explore the underlying mechanisms of behavioral homoplasy, a topic addressed in this review.

\section{Release Calls}

Female release calls are notably absent in the reticulated group within Clade 1 that includes $X$. boumbaensis [Tobias et al., 2014]. The presence of release calls in the related species $X$. laevis, $X$. poweri, and $X$. victorianus (Clade 1 ) as well as the 3 species comprising Clade 2 and their absence in the reticulated group in Clade 1 suggest that female release calling was lost from the reticulated group upon its divergence from its MRCA with the remainder of Clade $1 \sim 12$ mya [Tobias et al., 2014]. Loss of the female release call - though disadvantageous for a male's reproductive effort - might have contributed to hybridization by encouraging mating with the rare female on the cusp of receptivity. Female release calls within $X$. laevis, $X$. poweri, and X. victorianus (Clade 1 ), as well as within $X$. borealis, X. muelleri, and the undescribed species in Clade 2 are very similar in sound frequency but not in IPI [Tobias et al., 2014].

\section{Sex Differences in Call Characters across Species}

In the reticulated group of Clade 1, sex differences in vocal behaviors are extreme: males produce advertisement and release calls, but females do not produce release calls. Male advertisement calls are typically rapid (7-30 ms IPI), temporally complex (biphasic-type), and are intensity-modulated. Male release calls are rapid (i.e. 18-20 ms IPI) but temporally simple (monophasic). The exception is $X$. boumbaensis in which both the click-type advertisement call (IPI $>1,000 \mathrm{~ms})$ and the release call $(\sim 45 \mathrm{~ms}$ IPI) are slow relative to other species in the group. In the non-reticulated portion of the clade - X. laevis, X. poweri and $X$. victorianus - male advertisement calls also contain short IPIs, are temporally complex (biphasic- or bursttype), and are intensity-modulated. Male release calls are rapid and monophasic. Female release calls are significantly slower (100-200 ms IPI) and monophasic (fig. 3).

In Clade 2, differences between male and female call pulse rates are more modest, relative to differences in Clade 1. For example, male and female $X$. borealis release call IPIs average $\sim 40 \mathrm{~ms}$ and are not significantly different from one another (fig. 3) [Tobias et al., 2014]. In X. laevis (Clade 1) in contrast, release call IPIs are typically

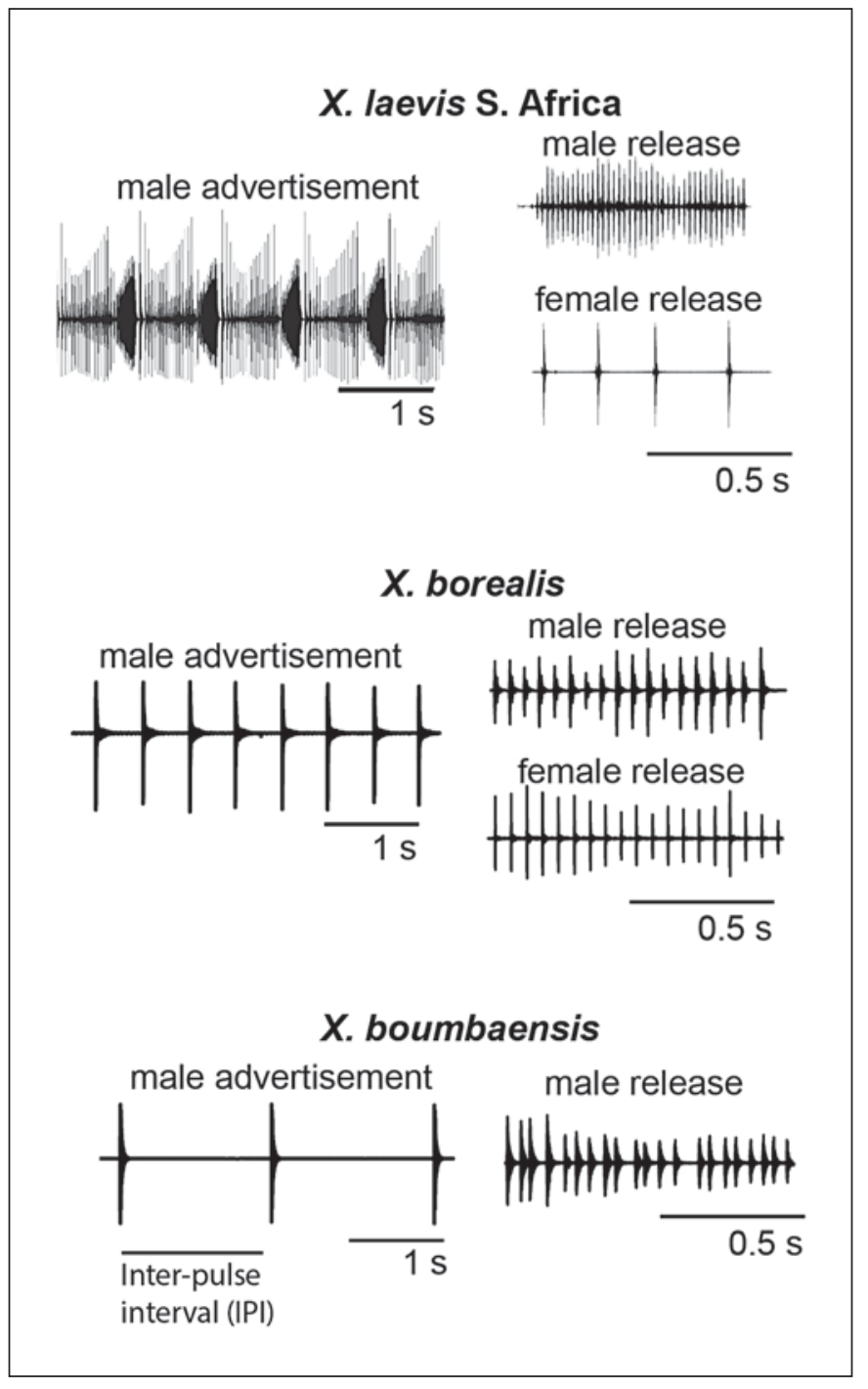

Fig. 3. Advertisement and release calls from X. laevis, X. boumbaensis, and $X$. borealis illustrate diversity in vocal sex differences in call complexity and IPI. In X. laevis (top), male advertisement and release call IPIs exceed those of females, and male calls are more temporally complex than female calls. X. boumbaensis (middle) and $X$. borealis (bottom) have temporally simplified male advertisement calls, with long IPIs. X. boumbaensis vocal sex differences are extreme, because females do not produce release calls. $X$. borealis vocal sex differences are reduced, as female and male release call IPIs do not differ significantly. Adapted from Leininger et al. [2015].

$10 \%$ of the female values (fig. 3) [Tobias et al., 2014]. In Clade 2, male advertisement calls deviate from the usual pattern of rapid (short IPIs) bursts or trills. X. borealis and the undescribed tetraploid species produce click-type advertisement calls (where the call is a single sound pulse 
repeated at long intervals), and $X$. muelleri produces a burst-type advertisement call with 2 sound pulses per call. The long IPI vocal character for the click-type male advertisement call is shared with X. boumbaensis in Clade 1 , the only other click-type caller.

In summary, Xenopus vocal repertoires exhibit robust sex differences in call rapidity and complexity, with a few notable exceptions. Species in Clade $2-X$. borealis, an undescribed tetraploid species (' $X$. new tetraploid'), and $X$. muelleri - exhibit reduced vocal sex differences in call rapidity alongside simplified, slow male advertisement call patterns. X. boumbaensis in Clade 1 is another interesting case. Vocal dimorphism is extreme because females do not produce release calls. The male X. boumbaensis vocal repertoire has independently evolved to the simple click-type advertisement pattern and a slow male release call that are more similar to the distantly related species (X. borealis) than to those of its own closest relatives [Leininger and Kelley, 2013; Leininger et al., 2015].

Based on this survey of sex differences across species, we propose that a sexually dimorphic vocal repertoire is ancestral and that the reduced vocal sex difference found, for example, in the $X$. borealis repertoire is evolutionarily derived. Next, we review our recent work probing the physiological bases for vocal simplification and reduced sexual dimorphism using the isolated singing brain and larynx of $X$. borealis and X. boumbaensis.

\section{Vocal Mechanisms: Sex and Species Differences}

\section{Sound Production in Xenopus}

Unlike in terrestrial anurans, Xenopus sounds are both produced and heard underwater [Vigny, 1979]. The vocal organ has accordingly been modified for underwater sound production from the ancestral terrestrial form [Yager, 1992b]. The Xenopus larynx consists of a frame of hyaline cartilage, stiffened by anterior-posterior bilateral rods of thyrohyal cartilage and flanked by a bipennate muscle bilaterally. The bipennate muscles insert anteriorly into paired, normally apposed, disks of arytenoid cartilage. Sound pulses are produced by separation of these disks. A single sound pulse is produced each time the disks are pulled apart; holding the disks apart prevents subsequent sound pulse production [Yager, 1992b].

This mechanism of sound production is readily apparent in the isolated (ex vivo) larynx: electrical stimulation of the laryngeal motor nerve rootlet is sufficient to generate laryngeal muscle activity, disk movements, and actual sound pulses [Tobias and Kelley, 1987] (figs. 4B, 5F, 6C).

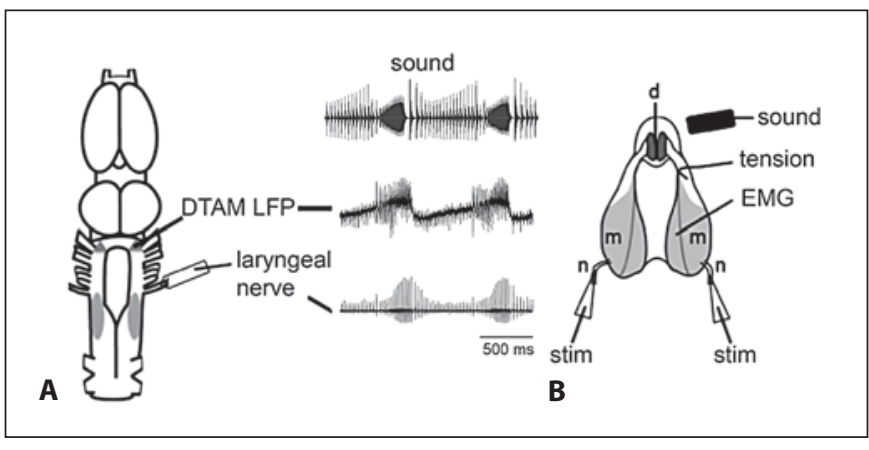

Fig. 4. Ex vivo preparations of the isolated brain and larynx. A Laryngeal nerve activity recorded from the isolated male $X$. laevis brain matches the pattern of sound pulses of the biphasic advertisement call. An LFP wave in the premotor nucleus DTAM coincides with fictive fast trill. B The isolated vocal organ (larynx) produces sound pulses (recorded with a hydrophone) when the laryngeal nerves ( $\mathrm{n}$ ) are stimulated, the muscles (m) contract, and the arytenoid disks (d) separate.

The ex vivo larynx is informative for understanding how vocalization parameters could be constrained peripherally, including the muscular constraints on sound pulse rate across Xenopus species and between the sexes. For example, $X$. laevis female laryngeal muscle, when stimulated via the nerve at male advertisement call IPIs, cannot contract and relax quickly enough to produce rapid trains of sound pulses, while male larynges can produce pulses at these stimulation intervals [Tobias and Kelley, 1987]. Sex differences in muscle contractile properties correspond to sex differences in laryngeal muscle fiber types: the male X. laevis laryngeal muscle is composed entirely of fast-twitch muscle fibers, while the female laryngeal muscle is composed of a mixture of fast-twitch and slow-twitch fibers [Sassoon et al., 1987]. The adult male laryngeal muscle expresses an embryonic, laryngeal-specific, myosin heavychain gene (laryngeal myosin; $L M$ ) in all muscle fibers [Catz et al., 1992]. In females, $L M$ is expressed only in some muscle fibers, which are fast-twitch [Catz et al., 1992].

Male laryngeal muscle activity increases over the course of a stimulus train delivered to the laryngeal nerve, due to the short-term strengthening of the weak laryngeal synapse with use (potentiation) [Tobias et al., 1995]. In females, the synapse is strong and does not potentiate [Ruel et al., 1998]. These synaptic sex differences contribute to the increasing intensity of sound pulses during a call (intensity modulation) in male calls and the ability of the female larynx to contract at slow rates of nerve activity without potentiation. 


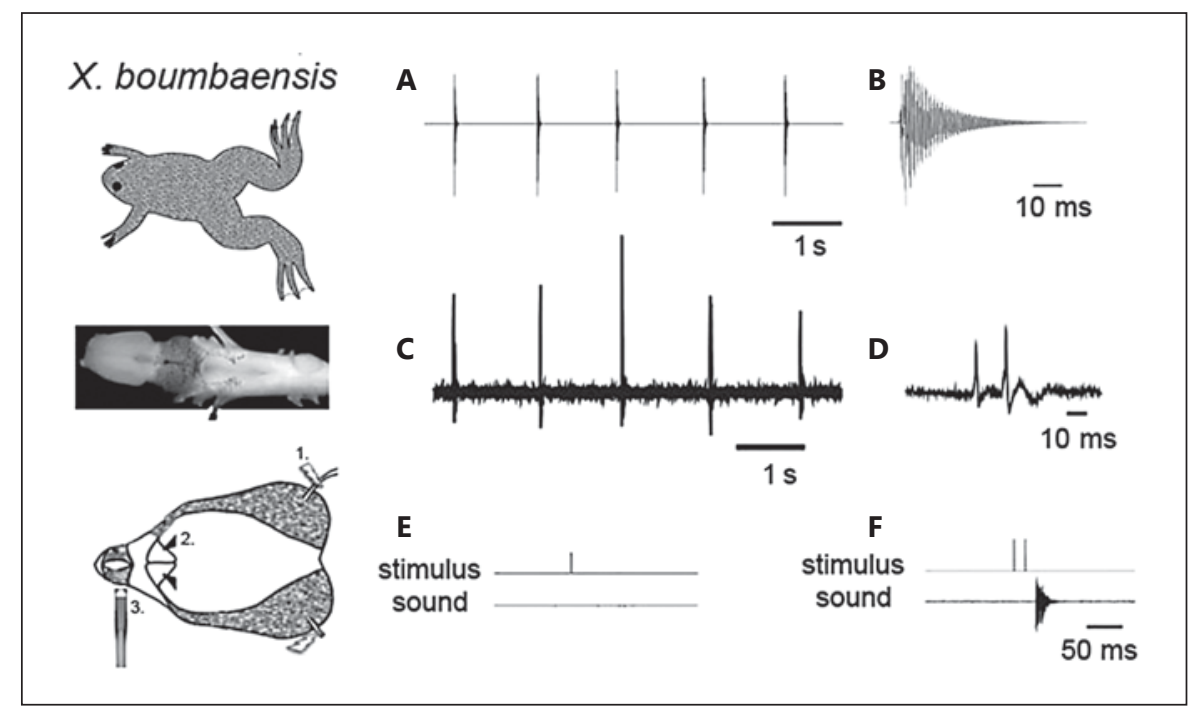

Fig. 5. Physiological bases of advertisement call production in $X$. boumbaensis. On the left, the preparation is indicated. The upper panel illustrates the calls; the middle panels show recordings from the laryngeal nerve (arrowhead) as it exits the brain; the lower panel illustrates results of electrically stimulating the laryngeal nerve (1.) and recording sounds (3.) produced by movements of the arytenoid disks (2.). A Sound oscillogram of the male X. boumbaensis advertisement call. The call is a series of sound pulses repeated at long (>1 s) intervals. B Enlargement of a single sound pulse.

\section{The Hindbrain Vocal Pattern Generator}

The laryngeal muscles receive input from neural circuits in the hindbrain via the laryngeal nerve which includes the axons of vocal motor neurons located in cranial nerve IX-X (the vocal motor nucleus, VMN). Activity in the laryngeal nerve comprises compound action potentials (CAPs) representing the activity of many motor neurons (fig. 4A). In X. laevis, the VPG includes bilateral nuclei: the VMN in the caudal hindbrain and the premotor nucleus DTAM (used as a proper name) in the rostral hindbrain. The VMN includes, in addition to vocal motor neurons [Kelley, 1980], motor neurons innervating the glottis [Tobias and Kelley, 1987; Zornik and Kelley, 2007], neurons that connect the VMN to the contralateral VMN, and neurons that project rostrally to DTAM [Zornik and Kelley, 2007]. Male and female laryngeal motor neurons differ in size [Simpson et al., 1986; Potter et al., 2005], in the prominence of several ion currents [Yamaguchi et al., 2003], and in the synchronicity of action potentials that comprise CAPs (fig. 6E, F) [Yamaguchi and Kelley, 2000]. Models suggest that individual motor neuron action potentials that comprise these differences contribute to the precision and rapidity of
C 5-HT-evoked laryngeal nerve recordings from the isolated $X$. boumbaensis brain reveal neural activity repeated at call intervals (compare to A). D Enlargement of one instance of neural activity in C. Nerve activity is composed of compound action potential doublets. E Single stimuli delivered to the laryngeal nerve rootlets are not sufficient to cause sound pulse production. F Doublet stimuli approximating the temporal parameters of the compound action potential doublets is sufficient to produce a single sound pulse. Adapted from Leininger and Kelley [2013].

motor output - and resultant sound pulse trills - specific to males [Yamaguchi et al., 2003].

Also of particular interest is a group of neurons in DTAM whose activity matches the fast trill portion of the male advertisement call [Zornik and Yamaguchi, 2012]. These 'fast trill neurons' exhibit an endogenous rhythmicity that sets the duration of the fast trill and thus the duration of the entire call (fast and slow trill) and also fire at intervals that approximate the sound IPIs in the fast trill of the advertisement call. Whether and how these neurons are tuned to species-specific firing rates is not known.

In X. laevis, recordings from the laryngeal nerve of calling frogs reveal that each CAP corresponds to one sound pulse within the call [Yamaguchi and Kelley, 2000]. The isolated brain, in response to exogenous serotonin application, produces CAP patterns that resemble those recorded in vivo (fig. 4A) [Rhodes et al., 2007]. This close correspondence holds both for the complex biphasic male advertisement call as well as for the simpler male and female release calls. VPG output also reflects additional call features such as intensity modulation: for example, progressive recruitment of laryngeal motor neu- 
Fig. 6. Physiological bases of advertisement call production in $X$. borealis. A Sound oscillogram of the male $X$. borealis advertisement call. The call is a series of sound pulses repeated at $\sim 450$-ms intervals. B 5-HTevoked laryngeal nerve recordings from the isolated $X$. borealis brain reveal neural activity repeated at intervals similar to advertisement call sound pulse intervals (compare to A). C In the isolated $X$. borealis male larynx, a single laryngeal nerve stimulation (1.) elicits a laryngeal electromyogram (2.), which causes one laryngeal muscle contraction (measured by tension recordings, 3.) resulting in a single sound pulse (4.). D A single CAP recorded from the laryngeal nerve in a male $X$. borealis brain. $X$. borealis male CAPs more closely resemble the asynchronous $X$. laevis female CAPs (E) than the highly synchronous $X$. laevis male CAPs (F). A-D Adapted from Leininger and Kelley [2013]. E, F Adapted from Yamaguchi and Kelley [2000].
A

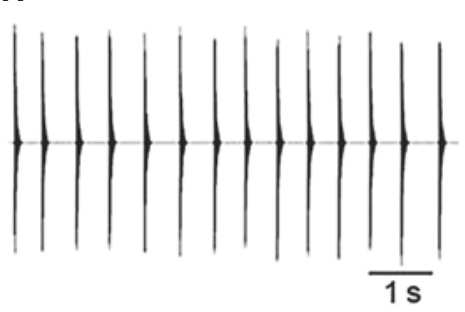

B

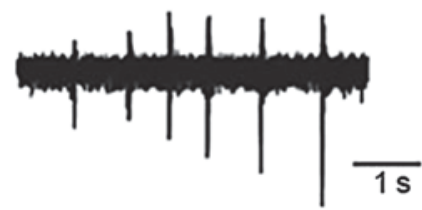

C
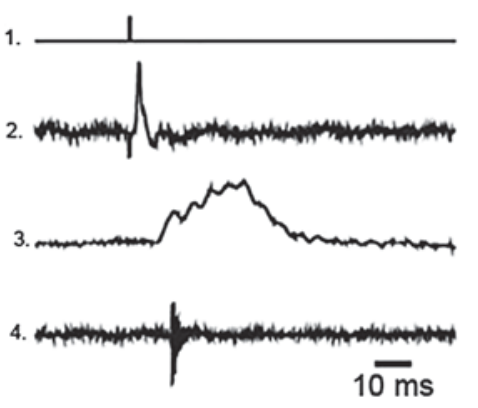

$X$. borealis
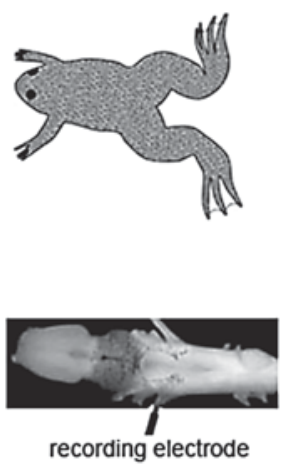

X. laevis
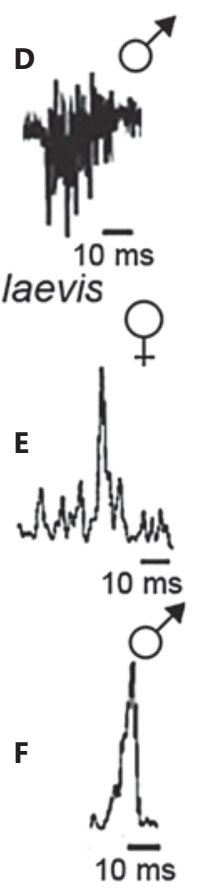

rons results in progressive increases in CAP amplitude on the laryngeal nerve, mirroring the sound pulse intensity increases seen in actual calls (fig. 4A) [Rhodes et al., 2007].

\section{Sexual Differentiation of the VPG and the Larynx}

A suite of sexually differentiated cellular and molecular features within the X. laevis VPG and larynx shape sex-specific vocalizations [reviewed in Zornik and Kelley, 2011]. In this species, sexually dimorphic neural and muscular mechanisms arise from a sexually monomorphic juvenile state in response to circulating gonadal steroid hormones [Kelley, 1996]. The juvenile state can resemble either the adult male or female state, and the effective hormones include gonadal androgens and estrogens. While hormones typically act on the developing organism to organize sex differences, the adult vocal hindbrain and larynx retain sensitivity to gonadal steroids into adulthood [Potter et al., 2005].

The sex difference in the size of laryngeal motor neurons reflects the action of gonadal androgens: many neu- rons in the $X$. laevis VMN express androgen receptor, as do neurons in DTAM [Kelley, 1980; Perez et al., 1996]. The sex difference in strength of the laryngeal synapse is due to gonadal estrogen [Tobias et al., 1995]. The estrogen receptor is expressed in laryngeal muscle but not in motor neurons, so developmental strengthening presumably reflects a retrograde signal from muscle to motor neuron [Wu et al., 2003].

\section{Convergent Evolution of Temporally Simplified Advertisement Calls: VPG and Laryngeal Mechanisms}

Species-specific mechanisms for Xenopus vocalization are less well understood. To probe the neural and muscular bases of species differences, we have studied $X$. borealis and $X$. boumbaensis, species with evolutionarily derived and temporally simplified male advertisement calls and, in the case of $X$. borealis, reduced vocal sex differences [Leininger and Kelley, 2013; Leininger et al., 
2015]. Below, we review neural and laryngeal mechanisms of vocal production in males and females of these species.

\section{Modifications of the VPG in X. boumbaensis}

$X$. boumbaensis produces male advertisement calls consisting of single sound pulses repeated at long intervals (fig. 5A, B). A parsimony analysis suggests that this clicktype call is derived from a more complex burst-type call (fig. 2) [Tobias et al., 2011]. What modifications in the VPG or larynx accompanied this change in vocal pattern?

We recorded fictive calling - vocal nerve activity from isolated male brains of $X$. boumbaensis. The output of the VPG in X. boumbaensis is an ancestral burst-type pattern consisting of repeated 2-CAP bursts of nerve activity (fig. 5D) [Leininger and Kelley, 2013]. In the isolated larynx, stimulus doublets approximating the CAP pattern produce a single sound pulse; single stimuli are ineffective at producing a sound pulse (fig. 5E, F). The vocal synapse thus requires potentiation to produce muscle contractions sufficient for sound pulse production. These findings indicate that the VPG of $X$. boumbaensis has been modified to produce the shortest possible burst of nerve activity (2 CAPs) required for sound production. The rapid CAP doublets produced by the VPG are separated by longer intervals, accounting for the long IPIs in the actual call. Male laryngeal muscle fibers are of the fasttwitch type and, after initial potentiation, capable of following longer bursts of rapid stimuli [Leininger et al., 2015]. However, the $X$. boumbaensis VPG does not generate prolonged, rapid bursts of activity.

What is the neurophysiological basis of call duration, and how might these mechanisms contribute to call evolution? In X. laevis, call duration is determined by activity in the most rostral component of the VPG, the nucleus DTAM [Zornik et al., 2010]. The fast trill phase coincides with a local field potential (LFP) wave in DTAM (fig. 4A), which requires the NMDA-type of glutamate receptor [Zornik et al., 2010]. Phasic activity, which correlates with VMN action potentials, rides on this wave. A modification in the activity of DTAM is thus the most likely locus for the evolutionary simplification of advertisement calls in X. boumbaensis. Reducing the duration of the LFP with shortened phasic activity could account for vocal simplification. Modifications associated with the NMDA receptor are thus candidate contributors to the VPG modification in $X$. boumbaensis responsible for call simplification.

In summary, the simplified advertisement call of $X$. boumbaensis is most likely due to a small alteration in the
VPG, namely a shorter duration of the DTAM LFP. The character states of other components of the vocal production system, such as laryngeal muscle fiber type, are identical to that of X. laevis [Sassoon et al., 1987] and S. tropicalis [Baur et al., 2008] and thus appear broadly conserved.

\section{Modifications of VPG and Laryngeal Muscle Fibers in}

\section{$X$. borealis}

$X$. borealis also produces male advertisement calls consisting of single sound pulses repeated at long intervals (fig. 6A) [Yager, 1992a; Tobias et al., 2011]. Are the modifications in the VPG and/or larynx that accompanied this change in vocal pattern the same as those seen in $X$. boumbaensis?

Recordings of fictive calling from isolated male brains in $X$. borealis reveal that the output of the VPG is a single CAP (fig. 6B, D). In the isolated larynx (fig. 6C), a single stimulus delivered to the laryngeal nerve (fig. $6 \mathrm{C} 1$ ) results in a robust muscle electromyogram (fig. 6C2), a tension transient recorded at the tendon (fig. 6C3) and a single sound pulse (fig. 6C4). No potentiation is required for muscle contraction. X. borealis laryngeal muscle contains a mix of fast- and slow-twitch fibers in both sexes [Leininger et al., 2015]. These neural and muscular features support production of sound pulses at slow, but not rapid, rates.

Thus, in contrast to X. boumbaensis, in X. borealis both the VPG output and laryngeal sound production are simplified. Many features of the VPG and vocal organ of male $X$. borealis resemble those of female (rather than male) $X$. laevis: the output of the VPG, the asynchrony in action potentials that make up the CAPs (fig. 6D; compare to fig. $6 \mathrm{E}, \mathrm{F}$ ), no requirement for potentiation, and the mixed population of muscle fiber twitch types. This comparison suggests that the vocal system in this species is feminized relative to members of Clade 1, resulting in a loss of physiological and behavioral sex differences.

\section{Candidate Endocrine Factors in X. borealis}

Sex differences in the X. laevis vocal circuit are hormonally regulated (fig. 7) [reviewed in Zornik and Kelley, 2011]. Androgen alters the VPG in females to produce male-like features [Rhodes et al., 2007]. The simple and slow VPG output in male $X$. borealis could reflect a loss of sensitivity to androgen in DTAM, accounting for slow CAP rates, and/or in the laryngeal motor neurons, accounting for asynchronous CAPs.

Available electromyogram (EMG) data in $X$. borealis suggest a large population of strong laryngeal synapses in 


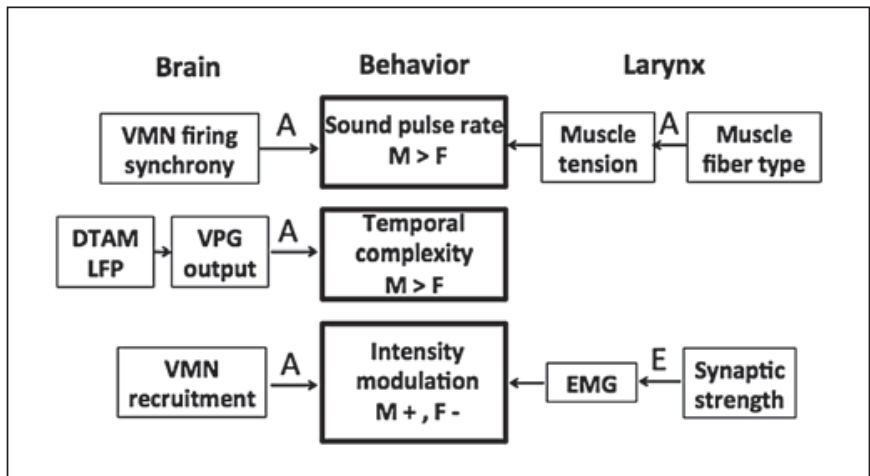

Fig. 7. A suite of hormonally controlled sex differences in the $X$. laevis vocal circuit contributes to vocal sex differences [reviewed in Zornik and Kelley, 2011]. Some behavioral traits - for example, sound pulse rate - rely both on brain and laryngeal mechanisms. Sex differences masculinize or feminize from a default juvenile state, in response to either androgens (A) or estrogens (E). Losses of these mechanisms are candidates for vocal evolution in species with reduced vocal sex differences, such as $X$. borealis.

both sexes [Leininger et al., 2015]. The X. laevis laryngeal neuromuscular synapse is mostly strong in adult females but mostly weak in adult males. The X. laevis laryngeal synapse is initially weak in both sexes but strengthens in developing females due to ovarian estrogen secretion [Wu et al., 2003]. Strong laryngeal synapses in male $X$. borealis could thus reflect heightened sensitivity to low levels of estrogen or a general insensitivity of synaptic strength to estrogen in both sexes.

$X$. borealis does not exhibit the sexually dimorphic laryngeal muscle fiber type seen in X. laevis, X. boumbaensis, and S. tropicalis; instead, both sexes exhibit a female-like mixture of fast- and slow-twitch fibers [Leininger et al., 2015]. The juvenile state of laryngeal muscle fiber in developing $X$. laevis [Sassoon et al., 1987] and $S$. tropicalis [Baur et al., 2008] is female-like. In developing male $X$. laevis and S. tropicalis, slow-twitch laryngeal fibers convert to fast-twitch due to gonadal androgen secretion [Tobias et al., 1991; Baur et al., 2008]. In X. laevis, androgen exposure prompts a population of myoblasts expressing the fast-twitch $L M$ gene to fuse with existing fibers [Nasipak and Kelley, 2012].

Evolutionary comparisons of fiber data from species assayed thus far suggest that sexually dimorphic laryngeal muscle fiber types are the ancestral state, present in X. laevis [Sassoon et al., 1987], S. tropicalis [Baur et al., 2008], X. boumbaensis [Leininger et al., 2015], and X. amieti [K. Kitayama, unpubl. data], but not in in X. borealis [Leininger et al., 2015]. X. borealis may thus have lost the an- cestral androgen-controlled developmental program for fiber type masculinization. This change could be due to deletion of the myoblast population, or loss of the androgen-driven program for proliferation and fusion. Overall, laryngeal size and muscle fiber number remain sexually dimorphic in X. borealis [Leininger et al., 2015], as is the case with X. laevis [Sassoon and Kelley, 1986], X. boumbaensis [Leininger et al., 2015], and S. tropicalis [Baur et al., 2008]. Therefore, we propose that these aspects of masculinization are conserved [Leininger et al., 2015].

In summary, we are beginning to understand the neural and muscular mechanisms responsible for speciesspecific vocalizations in Xenopus, both in terms of differences across species within a sex and with respect to sex differences across species. Of future interest is whether the VPG and vocal muscle differences described here are present in other species in Clade 2, which also exhibit atypically simplified male advertisement calls accompanying reduced vocal sex differences.

\section{Anuran Vocal Production}

Pipid frogs (including the subfamily Xenopodinae) evolved from terrestrial ancestors [Zhang et al., 2013]. We thus might expect shared features of vocal production between terrestrial and aquatic species, and this is the case. We might also expect differences that evolved in response to the shift to an aquatic habitat, and this is also true.

\section{Peripheral Mechanisms}

In terrestrial frogs, sound production is typically initiated and terminated by the activity of intrinsic laryngeal muscles and powered by airflow within the vocal tract. Schmidt [1965, 1972] observed movements of laryngeal and glottal muscles during male release calling (Rana) and advertisement calling ( Hyla) via a window created by unilateral enucleation. EMGs were recorded from the intrinsic laryngeal muscles. Although replacement of air in the lungs was required for vocalization, movements of the vocal muscles and EMG activity were not simply due to airflow as they occurred when the larynx was isolated from the respiratory tract. Calls instead reflected active central nervous system (CNS) control of laryngeal muscles. Martin and Gans [1972] confirmed and extended Schmidt's findings using male release calling in Bufo valliceps. Preparation for sound production required contraction of body wall muscles that compress the lungs. The sound pulses themselves, however, were controlled by relaxation and contraction of the laryngeal dilator and constrictor muscles. 
More recent studies have focused on the spectral features of sounds produced by airflow through the vocal tract either excised or in situ. While the complex, nonlinear spectral features of the ultrasonic calls in Amolops tormotus can be recreated by forcing air under pressure through the larynx [Suthers et al., 2006], in 3 other (nonultrasonic) species, this procedure produces a wider range of frequencies than those of actual calls [Gridi-Papp, 2014]. In actual calls, whether the mouth was open or closed (advertisement vs. distress calls) strongly influenced relative frequency intensities. Detailed mechanisms for frequency modulation have not been identified. In túngara frogs (Engystomops -previously Physalaemus-pustulosus) the production of a 'second voice' (the chuck that follows the whine) has been tied to a particular morphological feature: the fibrous mass [Gridi-Papp et al., 2006]. How the whine is produced has not yet been described in physiological detail.

The temporal features of advertisement and release calls are thus actively controlled by the CNS via innervation of intrinsic laryngeal muscles in both terrestrial and aquatic anurans. The spectral features of calls depend on the vocal tract in both groups. The major difference between terrestrial anurans and frogs of the subfamily Xenopodinae is the importance of airflow within the vocal tract: essential to powering sounds and shaping sound frequencies in terrestrial but not aquatic anurans. Glottal activity also differs in the 2 groups. In some terrestrial anurans the glottis opens and closes (pulses) during sound production [Schmidt, 1972]. In Xenopus, the glottis gates airflow between the buccal cavity and the lungs, and glottal muscles do not contract during call production [Zornik and Kelley, 2008]. The excised larynx can produce audible sounds without air movement [Tobias and Kelley, 1987], because the sound production mechanism simply involves movements of the arytenoid disks produced by laryngeal muscle contractions. The arytenoid cartilages also move during sound production in terrestrial anurans. For example, they vibrate during vocalization as a passive response to airflow and are moved out of the airstream by laryngeal muscles to terminate a sound pulse [Martin and Gans, 1972]. It would thus appear that while the shift to an aquatic environment resulted in modification of the Xenopus larynx, many shared components of sound production were retained from the ancestral state.

\section{Central Mechanisms}

Anurans are the only group in which a laryngeallybased hindbrain pattern generator has been identified. During electrically-evoked advertisement calling in Rana pipiens, Robert Schmidt [1974] recorded patterned neural activity from the hindbrain in the vicinity of the fifth cranial nerve. Lesion of this region blocked evoked calling, and stimulation evoked calling-associated laryngeal movements even in the isolated hindbrain. Schmidt went on to identify 2 independent hindbrain oscillators that influence calling in Rana pipiens: the pretrigeminal nucleus (PTN) and the classical pulmonary respiratory generator [Schmidt, 1992]. During the expiratory phase of respiration, activity corresponding to glottal opening, closure of the vocal cords, air movement past the cords, and a sound pulse could be recorded from the isolated brain in response to electrical stimulation of the forebrain.

DTAM closely resembles the PTN in terrestrial anurans and the nuclei are likely homologs [Schmidt, 1992]. However, in Xenopus we have not yet observed rhythmic activity in the pulmonary pattern generator, which is coextensive with the VMN in Schmidt's studies. Producing sounds underwater decouples vocalization from respiration, and this portion of the anuran VPG may have been lost in Xenopodinae.

\section{Evolutionary Origins of Vocal Production}

Mechanisms in Vertebrates

In vertebrates, the mechanisms and organs of vocal production are highly variable and include wing movements [Bostwick and Prum, 2005], swim bladder contractions [Fine, 2012], vibration of syringeal membranes [Elemans, 2014], as well as the laryngeal mechanisms common to mammals, basal reptilians, and frogs [Riede et al., 2015].

Mammals and birds differ from anurans in that vocalizations are produced during actual respiration (typically expiration) rather than just during air movement within the vocal tract. Whether the link between breathing and sound production is a conserved feature or has instead evolved independently is not known. Nevertheless, the interposition of sound-producing elements within the respiratory tracts in these groups as well as in frogs suggests that the neural circuitry for breathing and respiration might have shared evolutionary origins; i.e. a portion of the respiratory circuit may have been exapted for vocalization. If so, it might be possible to identify these shared features in chordates, such as the lamprey.

In lampreys, gill-based inspiration is passive but expiration is driven by rhythmic activity in the paratrigeminal respiratory group (pTRG) [Bongianni et al., 2014]. The pTRG is located in the pons, immediately ventral to the cerebellum, produces rhythmic activity in a fictive breathing preparation, and projects directly to N. IX-X. It has 
been proposed [Bongianni et al., 2014] that the pTRG is an evolutionary precursor of the preBötzinger nucleus, ventro-posterior to N. IX-X, that is an important component of the respiratory circuitry [Gray et al., 1999]. However, the pTRG is located in the rostral pons and the preBötzinger nucleus is in the caudal medulla, and their connectivities differ substantially. The pTRG in lampreys does share location, connectivity, and rhythmogenesis with DTAM/PTN and could be homologous. Ongoing examination of transcription factors and other molecular markers [Sweeney and Kelley, 2014] may be helpful in establishing if these nuclei are homologous. If so, links between breathing and calling would have been maintained in Xenopus. Further modification, such as the inhibition of glottal motor neurons [Zornik and Kelley, 2008], might then have accompanied the transition to the underwater habitat.

\section{Summary}

Xenopus provides an informative model system for investigating the biological bases of behavior and its evolution, particularly with respect to sexually dimorphic be- haviors. In X. laevis, the rich array of sex differences in the vocal circuit that map to sexually dimorphic behavioral features provides candidate mechanisms for vocal evolution. Losses of sexually differentiated neural and neuromuscular features might explain male vocal simplification and reduced sex differences. More subtle species differences, such as differences in sound IPI within the burst-type temporal structure, may rely on more minor changes in nervous system and muscular function and have yet to be elucidated. Integrating recent approaches in genomics with our understanding of the molecular bases of behavior in model Xenopus species will be helpful for uncovering molecular bases of species differences and their regulation.

\section{Acknowledgments}

We thank Charlotte Barkan for providing recordings shown in figure 4 . We also thank 3 anonymous reviewers for constructive feedback on the manuscript and Ben Evans for helpful editorial suggestions.

The research described here was supported by NS23684 (D.B.K.), research funds associated with the Weintraub Chair (D.B.K.), the Columbia Science Fellows Program, and St. Mary's College of Maryland (E.C.L.).

\section{References}

Baur LA, Nasipak BT, Kelley DB: Sexually differentiated, androgen-regulated, larynx-specific myosin heavy chain isoforms in Xenopus tropicalis and Xenopus laevis. Dev Genes Evol 218:371-379 (2008).

Bewick AJ, Anderson DW, Evans BJ: Evolution of the closely related, sex-related genes $D M-W$ and DMRT1 in African clawed frogs (Xenopus). Evolution 65:698-712 (2011).

Bongianni F, Mutolo D, Cinelli E, Pantaleo T: Neural mechanisms underlying respiratory rhythm generation in the lamprey. Respiratory Physiol Neurobiol, DOI: 10.1016/j. resp.2014.09.003. [Epub ahead of print] (2014).

Bostwick KS, Prum RO: Courting bird sings with stridulating wing feathers. Science 309:736736 (2005).

-Catz DS, Fischer LM, Moschella MC, Tobias ML, Kelley, DB: Sexually dimorphic expression of a laryngeal-specific, androgen-regulated myosin heavy chain gene during Xenopus laevis development. Dev Biol 152:366-376 (1992).

Elemans CP: The singer and the song: the neuromechanics of avian sound production. Curr Opin Neurobiol 28:172-178 (2014).

Elliott TM, Kelley DB: Male discrimination of receptive and unreceptive female calls by temporal features. J Exp Biol 210:2836-2842 (2007).
Evans BJ: Ancestry influences the fate of duplicated genes millions of years after duplication in allopolyploid clawed frogs (Xenopus). Genetics 176:1119-1130 (2007).

Evans BJ: Genome evolution and speciation genetics of clawed frogs (Xenopus and Silurana). Front Biosci 13:4687-4706 (2008).

Evans BJ, Kelley DB, Tinsley RC, Melnick DJ, Cannatella DC: A mitochondrial DNA phylogeny of clawed frogs: phylogeography on sub-Saharan Africa and implications for polyploid evolution. Mol Phylogenet Evol 33:197213 (2004).

-Evans BJ, Kelley DB, Melnick DJ, Cannatella DC: Evolution of $R A G-1$ in polyploid clawed frogs. Mol Biol Evol 22:1193-1207 (2005).

- Fine ML: Swimbladder sound production: the forced response versus the resonant bubble. Bioacoustics 21:5-7 (2012).

Gerhardt HC, Huber F: Acoustic Communication in Insects and Anurans, ed 1 (University of Chicago Press, Chicago 2002).

Gray PA, Rekling JC, Bocchiaro CM, Feldman D: Modulation of respiratory frequency by peptidergic input to rhythmogenic neurons in the preBötzinger complex. Science 286:15661568 (1999).
Gridi-Papp M: Is the frequency content of the calls in north American treefrogs limited by their larynges? Int J Evol Biol, DOI: 10.1155/2014/198069 (2014).

Gridi-Papp M, Rand AS, Ryan MJ: Animal communication: complex call production in the túngara frog. Nature 441:38-38 (2006).

Hellsten U, Harland RM, Gilchrist MJ, Hendrix D, Jurka VK, et al: The genome of the Western clawed frog Xenopus tropicalis. Science 328: 633-636 (2010).

Kelley DB: Auditory and vocal nuclei of frog brain concentrate sex hormones. Science 207:553555 (1980).

Kelley DB: Sexual differentiation in Xenopus laevis, in Kobel HR, Tinsley RC (eds): The Biology of Xenopus, pp 143-176 (Oxford University Press, Oxford 1996).

Kobel HR, Loumont C, Tinsley RC: The extant species, in Kobel HR, Tinsley RC (eds): The Biology of Xenopus, pp 9-33 (Oxford University Press, Oxford 1996).

Leininger EC, Kelley DB: Distinct neural and neuromuscular strategies underlie independent evolution of simplified advertisement calls. Proc Biol Sci, DOI: 10.1098/rspb.2012.2639 (2013). 
Leininger EC, Kitayama K, Kelley DB: Speciesspecific loss of sexual dimorphism in vocal effectors accompanies vocal simplification in African clawed frogs (Xenopus). J Exp Biol 218:849-857 (2015).

Martin WF, Gans C: Muscular control of the vocal tract during release signaling in the toad Bufo valliceps. J Morphol 137:1-27 (1972).

- Nasipak BT, Kelley DB: Developing laryngeal muscle of Xenopus laevis as a model system: androgen-driven myogenesis controls fiber type transformation. Dev Neurobiol 72:664675 (2012)

Picker MD: Hormonal induction of the aquatic phonotactic response of Xenopus. Behaviour 84:74-90 (1983).

Pérez J, Cohen MA, Kelley DB: Androgen receptor mRNA expression in Xenopus laevis CNS: sexual dimorphism and regulation in laryngeal motor nucleus. J Neurobiol 30:556-568 (1996).

Potter KA, Bose T, Yamaguchi A: Androgen-induced vocal transformation in adult female African clawed frogs. J Neurophysiol 94:415428 (2005).

Rhodes HJ, Yu HJ, Yamaguchi A: Xenopus vocalizations are controlled by a sexually differentiated central pattern generator. J Neurosci 27:1485-1497 (2007)

Riede T, Li Z, Tokuda IT, Farmer CG: Functional morphology of the Alligator mississippiensis larynx and implications for vocal production. J Exp Biol 218:991-998 (2015).

Ruel TD, Kelley DB, Tobias ML: Facilitation at the sexually differentiated laryngeal synapse of Xenopus laevis. J Comp Physiol 182:35-42 (1998).

-Sassoon DA, Kelley DB: The sexually dimorphic larynx of Xenopus laevis: development and androgen regulation. Am J Anat 177:457-472 (1986).

-Sassoon DA, Gray G, Kelley DB: Androgen regulation of muscle fiber type in the sexually dimorphic larynx of Xenopus laevis. J Neurosci 7:3198-320 (1987).

- Schmidt RS: Larynx control and call production in frogs. Copeia 2:143-147 (1965).

Schmidt RS: Action of intrinsic laryngeal muscles during release calling in leopard frog. J Exp Zool 181:233-243 (1972).
Schmidt RS: Neural correlates of frog calling-trigeminal tegmentum. J Comp Physiol 92:229254 (1974).

Schmidt RS: Neural correlates of frog calling: production by two semi-independent generators. Behav Brain Res 50:17-30 (1992).

Simpson HB, Tobias ML, Kelley DB: Origin and identification of fibers in the cranial nerve IX-X complex of Xenopus laevis: Lucifer Yellow backfills in vitro. J Comp Neurol 244: 430-444 (1986).

Suthers RA, Narins P, Lin WY, Schnitzler HU, Denzinger A, et al: Voices of the dead: complex nonlinear vocal signals from the larynx of an ultrasonic frog. J Exp Biol 209:49844993 (2006).

Sweeney L, Kelley DB: Harnessing vocal patterns for social communication. Curr Opin Neurobiol 28:34-41 (2014).

Tobias ML, Kelley DB: Vocalizations of a sexually dimorphic isolated larynx: peripheral constraints on behavioral expression. J Neurosci 7:3191-3197 (1987)

Tobias ML, Marin ML, Kelley DB: Temporal constraints on androgen directed laryngeal masculinization in Xenopus laevis. Dev Biol 147: 260-270 (1991).

Tobias ML, Kelley DB, Ellisman M: A sex difference in synaptic efficacy at the laryngeal neuromuscular junction of Xenopus laevis. J Neurosci 15:1660-1668 (1995).

Tobias ML, Viswanathan S, Kelley DB: Rapping, a female receptive call, initiates male/female duets in the South African clawed frog. Proc Natl Acad Sci USA 95:1870-1875 (1998).

Tobias ML, O'Hagan R, Horng SH, Rand M, Kelley DB: Vocal communication between male Xenopus laevis; behavioral context and sexual state. Anim Behav 67:353-365 (2004).

Tobias ML, Corke A, Korsh J, Yin D, Kelley DB: Vocal competition in male Xenopus laevis. Behav Ecol Sociobiol 65:1791-1803 (2010).

Tobias ML, Kelley DB, Evans BJ: Evolution of advertisement calls in African clawed frogs. Behaviour 148:519-549 (2011).

Tobias MT, Korsh J, Kelley DB: Evolution of male and female release calls in African clawed frogs. Behaviour 151:1313-1334 (2014).
Vignal C, Kelley D: Significance of temporal and spectral acoustic cues for sexual recognition in Xenopus laevis. Proc Biol Sci 274:479-488 (2007).

Vigny C: The mating calls of 12 species and subspecies of the genus Xenopus (Amphibia: Anura). J Zool 188:103-122 (1979).

Wu KH, Tobias ML, Kelley DB: Estrogen receptor expression in laryngeal muscle in relation to estrogen dependent increases in synaptic strength. Neuroendocrinol 78:72-80 (2003).

Yager DD: Underwater acoustic communication in the African pipid frog Xenopus borealis. Bioacoustics 4:1-24 (1992a)

- Yager DD: A unique sound production mechanism in the pipid anuran Xenopus borealis. Zool J Linn Society 104:351-375 (1992b).

- Yamaguchi A, Kelley DB: Generating sexually differentiated vocal patterns: laryngeal nerve and EMG recordings from vocalizing male and female African clawed frogs (Xenopus laevis). J Neurosci 20:1559-1567 (2000).

- Yamaguchi A, Kaczmarek L, Kelley DB: Functional specialization of male and female motoneurons. J Neurosci 23:11568-11576 (2003).

-Zhang P, Liang D, Mao RL Hillis DM, Wake DB, Cannatella DC: Efficient sequencing of Anuran mtDNAs and a mitogenomic exploration of the phylogeny and evolution of frogs. Mol Biol Evol 30:1899-1915 (2013).

Zornik E, Kelley DB: Breathing and calling: neuronal networks in the Xenopus laevis hindbrain. J Comp Neurol 501:303-315 (2007).

Zornik E, Kelley DB: Regulation of respiratory and vocal motor pools in the isolated brain of Xenopus laevis. J Neurosci 28:612-621 (2008).

Zornik E, Kelley DB: Neuroendocrine basis for the hierarchical control of frog courtship vocalizations. Front Neuroendocrinol 32:353366 (2011)

Zornik E, Yamaguchi A: Coding rate and duration of vocalizations of the frog, Xenopus laevis. J Neurosci 32:12102-12114 (2012).

Zornik E, Katzen AW, Rhodes HJ, Yamaguchi A NMDAR-dependent control of call duration in Xenopus laevis. J Neurophysiol 103:35013515 (2010). 